\title{
In Situ Thermometry of a Cold Fermi Gas via Dephasing Impurities
}

\author{
Mark T. Mitchison $\odot,{ }^{1, *}$ Thomás Fogarty $\odot,{ }^{2}$ Giacomo Guarnieri®, ${ }^{1}$ Steve Campbellø, ${ }^{3}$ \\ Thomas Busch $\oplus^{2}$ and John Goold $\oplus^{1, \dagger}$ \\ ${ }^{1}$ School of Physics, Trinity College Dublin, College Green, Dublin 2, Ireland \\ ${ }^{2}$ Quantum Systems Unit, Okinawa Institute of Science and Technology Graduate University, \\ Onna, Okinawa 904-0495, Japan \\ ${ }^{3}$ School of Physics, University College Dublin, Belfield Dublin 4, Ireland
}

(Received 13 April 2020; accepted 27 July 2020; published 19 August 2020)

\begin{abstract}
The precise measurement of low temperatures is a challenging, important, and fundamental task for quantum science. In particular, in situ thermometry is highly desirable for cold atomic systems due to their potential for quantum simulation. Here, we demonstrate that the temperature of a noninteracting Fermi gas can be accurately inferred from the nonequilibrium dynamics of impurities immersed within it, using an interferometric protocol and established experimental methods. Adopting tools from the theory of quantum parameter estimation, we show that our proposed scheme achieves optimal precision in the relevant temperature regime for degenerate Fermi gases in current experiments. We also discover an intriguing trade-off between measurement time and thermometric precision that is controlled by the impurity-gas coupling, with weak coupling leading to the greatest sensitivities. This is explained as a consequence of the slow decoherence associated with the onset of the Anderson orthogonality catastrophe, which dominates the gas dynamics following its local interaction with the immersed impurity.
\end{abstract}

DOI: 10.1103/PhysRevLett.125.080402

Temperature measurements are crucial for many experiments using ultracold atomic gases, for example when calibrating quantum simulators $[1,2]$ or when determining equations of state $[3,4]$. Unfortunately, standard thermometry techniques such as time-of-flight or in situ absorption imaging are inherently destructive and involve integration over the line of sight [5]. A minimally disturbing method to probe local temperature profiles would be beneficial for numerous experimental scenarios of current interest, including thermalization dynamics after a quench [6-10] or energy transport between separate thermal reservoirs $[11,12]$. Further motivation is provided by recent progress in the preparation of homogeneous ultracold gases [13-19], whose constant density distribution does not carry information on temperature, thus rendering standard in situ thermometry techniques ineffective.

An appealing alternative method of in situ thermometry exploits impurity atoms as probes embedded within the ultracold gas [20-24]. The advantage of this approach is that a single atom can be confined to submicron length scales and its state is relatively easy to characterize. For example, temperature can be inferred by allowing the

Published by the American Physical Society under the terms of the Creative Commons Attribution 4.0 International license. Further distribution of this work must maintain attribution to the author(s) and the published article's title, journal citation, and DOI. impurities to equilibrate with the gas and then measuring their mean energy or a similar observable [25-27]. This method has proved useful in several recent experiments [28-34] but becomes challenging at low temperatures where equilibration is slow and the probe's energy levels must be finely tuned [26,35-40]. These limitations can be overcome by harnessing the probe's nonequilibrium dynamics for thermometry [25,41-46]. Perhaps the most extreme example is pure dephasing, where the energy of the probe is conserved and thus normal thermalization is completely suppressed. Nevertheless, coherences between the probe energy eigenstates can develop into correlations with the environment that are sensitive to temperature [47-50].

In this Letter, we apply this idea to address a longstanding challenge in cold-atom physics: namely, thermometry of degenerate Fermi gases [51,52]. Specifically, we propose to measure the temperature of an ultracold Fermi gas by observing the nonequilibrium dephasing dynamics of impurities immersed within it. We focus on a promising setup that has already been realized in the laboratory [53-55], where the gas atoms effectively interact only with the impurities and not with each other. In this setting, the Anderson orthogonality catastrophe (OC) $[56,57]$ imprints characteristic signatures on the decoherence dynamics of the impurity [58-61], which can be observed using Ramsey interferometry [54,62,63]. The optimal precision of our thermometry protocol can be evaluated in terms of the quantum Fisher information, 
and we reveal a tradeoff between measurement time and precision controlled by the impurity-gas interaction strength. Since this coupling can be experimentally tuned over a wide range of values by means of Feshbach resonances [64], our approach allows for precise in situ thermometry of homogeneous Fermi gases in the deeply degenerate regime.

Thermometry by qubit dephasing. - Let us begin with the general scenario of a two-level probe (qubit) $S$ undergoing pure dephasing induced by its environment $E$. The total Hamiltonian is $\hat{H}=\hat{H}_{S}+\hat{H}_{E}+\hat{H}_{I}$, where $\hat{H}_{I}$ is an interaction which satisfies $\left[\hat{H}_{S}, \hat{H}_{I}\right]=0$. We assume that the system is initially prepared in the product state $\hat{\rho}=|+\rangle\langle+| \otimes \hat{\rho}_{E}(T)$, where $\hat{\rho}_{E}(T)$ is a thermal state of the environment at temperature $T$ and $|+\rangle=(|0\rangle+$ $|1\rangle) / \sqrt{2}$ is an equal superposition of the qubit's energy eigenstates. The populations of these eigenstates are strictly conserved in time, while the qubit coherences decay according to the decoherence function

$$
v(t)=\operatorname{Tr}_{E}\left[e^{i \hat{H}_{1} t / \hbar} e^{-i \hat{H}_{0} t / \hbar} \hat{\rho}_{E}(T)\right],
$$

where $\hat{H}_{j}=\left\langle j\left|\hat{H}_{E}+\hat{H}_{I}\right| j\right\rangle$ is the Hamiltonian of the environment conditioned on the qubit eigenstate $j=0$, 1. In a frame rotating at the qubit precession frequency, the state of the qubit is given by $\hat{\rho}_{S}=\frac{1}{2}(1+\mathbf{v} \cdot \hat{\boldsymbol{\sigma}})$, where $\mathbf{v}=(\operatorname{Re}[v], \operatorname{Im}[v], 0)$ is the Bloch vector and $\hat{\boldsymbol{\sigma}}=$ $\left(\hat{\sigma}_{x}, \hat{\sigma}_{y}, \hat{\sigma}_{z}\right)$ are Pauli matrices.

The initial temperature of the gas parametrizes the probe state $\hat{\rho}_{S}(T)$ via the decoherence function in Eq. (1). If the dependence of $v(t)$ on $T$ is well understood, this temperature can therefore be inferred from the statistics of measurements made on a large ensemble of identically prepared probes. Any such temperature estimate carries an unavoidable uncertainty due to the random character of quantum measurement and the finite size of the ensemble. To find the optimal measurement that minimizes this uncertainty, we appeal to the theory of quantum parameter estimation [65-67].

In general, a measurement is described by a positive operator-valued measure $\{\hat{\Pi}(\xi)\}$ satisfying $\int d \xi \hat{\Pi}(\xi)=\mathbb{1}$, where $\xi$ labels the possible outcomes. Performing $N$ independent measurements on identical qubit preparations yields the random outcomes $\boldsymbol{\xi}=\left\{\xi_{1}, \xi_{2}, \ldots, \xi_{N}\right\}$, from which a temperature prediction is generated via an estimator function $T_{\text {est }}(\xi)$. We consider unbiased estimators with $\mathbb{E}\left[T_{\text {est }}\right]=T$, where

$\mathbb{E}\left[T_{\text {est }}\right]=\int d \xi_{1} \cdots \int d \xi_{N} p\left(\xi_{1} \mid T\right) \cdots p\left(\xi_{N} \mid T\right) T_{\text {est }}(\boldsymbol{\xi})$,

and $p(\xi \mid T)=\operatorname{Tr}\left[\hat{\Pi}(\xi) \hat{\rho}_{S}(T)\right]$. The expected uncertainty of the temperature estimate is quantified by $\Delta T^{2}=$ $\mathbb{E}\left[\left(T_{\text {est }}-T\right)^{2}\right]$ and the error of any unbiased estimator obeys the quantum Cramér-Rao bound $\Delta T^{2} \geq 1 / N \mathcal{F}_{T} \geq$
$1 / N \mathcal{F}_{T}^{Q}[68]$. Here, $\mathcal{F}_{T}$ is the Fisher information associated with the measurement,

$\mathcal{F}_{T}=\int d \xi p(\xi \mid T)\left(\frac{\partial \ln p(\xi \mid T)}{\partial T}\right)^{2}=\frac{1}{\left\langle\Delta \hat{X}^{2}\right\rangle}\left(\frac{\partial\langle\hat{X}\rangle}{\partial T}\right)^{2}$,

and the second equality holds for projective measurements on a two-level system, with $\langle\hat{X}\rangle$ and $\left\langle\Delta \hat{X}^{2}\right\rangle$ the mean and variance of the measured observable $\hat{X}$. The Fisher information of any positive operator-valued measure is bounded by the quantum Fisher information (QFI) $\mathcal{F}_{T}^{Q}=$ $\max _{\hat{X}} \mathcal{F}_{T}(\hat{X})=\mathcal{F}_{T}\left(\hat{\Lambda}_{T}\right)$ and the maximum is achieved by projective measurements of a specific observable: the symmetric logarithmic derivative (SLD), denoted by $\hat{\Lambda}_{T}$ [65]. We also define the quantum signal-to-noise ratio (QSNR) $\mathcal{Q}^{2}=T^{2} \mathcal{F}_{T}^{Q}$, which bounds the signal-to-noise ratio as $T / \Delta T \leq \sqrt{N} \mathcal{Q}$. Hence, $\mathcal{Q}$ quantifies the ultimate sensitivity limit of our impurity thermometer.

For a qubit probe, the QFI has a simple expression in terms of the Bloch vector [69], and for pure dephasing it can be conveniently written in polar coordinates using $v=$ $|v| e^{i \phi}$ as

$\mathcal{F}_{T}^{Q}=\frac{1}{1-|v|^{2}}\left(\frac{\partial|v|}{\partial T}\right)^{2}+|v|^{2}\left(\frac{\partial \phi}{\partial T}\right)^{2}=\mathcal{F}_{T}^{\|}+\mathcal{F}_{T}^{\perp}$.

The QFI comprises two terms, respectively corresponding to the Fisher information for measurements of $\hat{\sigma}_{\|}=$ $\cos (\phi) \hat{\sigma}_{x}+\sin (\phi) \hat{\sigma}_{y}$ and $\hat{\sigma}_{\perp}=\cos (\phi) \hat{\sigma}_{y}-\sin (\phi) \hat{\sigma}_{x}$, i.e., parallel and perpendicular to the Bloch vector of $\hat{\rho}_{S}(T)$. Neglecting irrelevant shift and scale factors, the SLD is given by

$\hat{\Lambda}_{T} \propto \cos (\varphi) \hat{\sigma}_{\|}+\sin (\varphi) \hat{\sigma}_{\perp}, \quad \tan (\varphi)=\frac{|v|(1-|v|)^{2} \partial_{T} \phi}{\partial_{T}|v|}$.

Since the SLD is optimal in the sense of the quantum Cramér-Rao bound, measuring $\hat{\Lambda}_{T}$ minimizes the uncertainty in the temperature estimate due to the finite number of samples. Note that the SLD is temperature dependent and thus some prior information on $T$ is assumed. In practice, measuring $\hat{\Lambda}_{T}$ requires an efficient prescription to evaluate $|v|, \phi$ and their temperature derivatives from an accurate theoretical model for $\hat{\rho}_{S}(T)$, as well as the ability to measure an arbitrary combination of $\hat{\sigma}_{x}$ and $\hat{\sigma}_{y}$.

Physical model.-From here on, we focus on a scenario realized in recent experiments [54], which satisfies the aforementioned desiderata for optimal thermometry. Here, the qubit comprises two spin states of an impurity immersed in a spin-polarized Fermi gas (see Fig. 1). We assume that the impurity is confined to the ground state of a species-selective potential so that its kinetic energy can be neglected. The only relevant collision process at low temperatures is $s$-wave scattering, which does not occur 

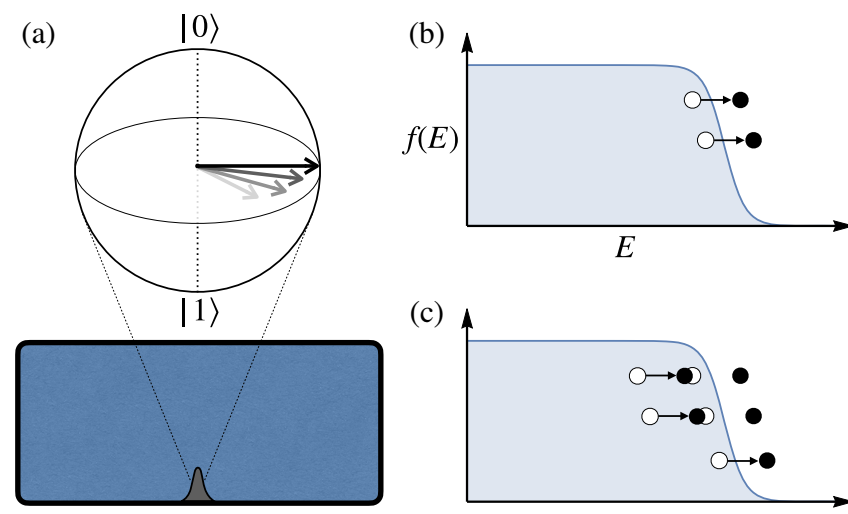

(c)

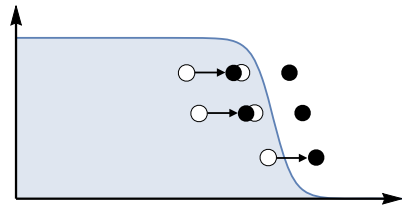

FIG. 1. Schematic depiction of the system. (a) A cold Fermi gas (blue) is perturbed by a localized impurity (gray) with two internal states that undergo pure dephasing. (b) Scattering from the impurity disturbs the atoms' initial equilibrium distribution $f(E)$. Pauli blocking restricts the resulting particle-hole excitations to a region near the Fermi surface. (c) The creation of holes eventually allows further scattering to generate excitations deep within the Fermi sea.

between identical fermions due to wave function antisymmetry. Therefore, the gas atoms do not interact with each other, while their coupling to the impurity is controlled by a spin-dependent $s$-wave scattering length. We assume that the impurity and the gas interact only when the impurity is in state $|1\rangle$, which can be achieved by tuning the scattering length for state $|0\rangle$ to zero via a Feshbach resonance [64].

We consider the following interferometric protocol. The gas is prepared in a thermal state with the impurity in the noninteracting state $|0\rangle$, leading to an initial density matrix $\hat{\rho}=|0\rangle\langle 0| \otimes \hat{\rho}_{E}(T)$. A $\pi / 2$ pulse then prepares the superposition state $|0\rangle \rightarrow|+\rangle$ and the system freely evolves for a time $t$, after which the qubit coherences are given by Eq. (1). Finally, a second $\pi / 2$ pulse is applied with a phase $\theta$ relative to the initial pulse and the qubit's energy is projectively measured, giving a result proportional to $\cos (\theta)\left\langle\hat{\sigma}_{x}\right\rangle+\sin (\theta)\left\langle\hat{\sigma}_{y}\right\rangle$ on average. Repeating this procedure $N$ times-or using $N$ independent impurities interacting with a single copy of the gas-yields the expectation value of any combination of $\hat{\sigma}_{x}$ and $\hat{\sigma}_{y}$, e.g., choosing $\theta=\phi+\varphi$ realizes a measurement of $\hat{\Lambda}_{T}$.

For a noninteracting gas, the decoherence function can be computed exactly using the Levitov formula $[70,71]$

$$
v(t)=\operatorname{det}\left[1-\hat{n}+\hat{n} e^{i \hat{h}_{0} t / \hbar} e^{-i \hat{h}_{1} t / \hbar}\right],
$$

where $\hat{h}_{1}$ and $\hat{h}_{0}$ are single-particle Hamiltonians describing atoms in the gas with or without the impurity present, respectively. The initial thermal distribution is described by $\hat{n}=\left(e^{\beta\left(\hat{h}_{0}-\mu\right)}+1\right)^{-1}$, where $\beta=1 / k_{B} T$ and $\mu$ is the chemical potential. In general, we have

$$
\begin{gathered}
\hat{h}_{0}=\frac{-\hbar^{2}}{2 m} \nabla^{2}+V_{\text {ext }}(\mathbf{r}), \\
\hat{h}_{1}=\hat{h}_{0}+V_{\text {imp }}(\mathbf{r}),
\end{gathered}
$$

where $m$ is the atomic mass, $V_{\text {ext }}(\mathbf{r})$ is an external potential, $V_{\text {imp }}(\mathbf{r})=\int d \mathbf{r}^{\prime} V_{\text {int }}\left(\mathbf{r}-\mathbf{r}^{\prime}\right)\left|\chi\left(\mathbf{r}^{\prime}\right)\right|^{2}$ is the scattering potential generated by a static impurity with wave function $\chi(\mathbf{r})$, and $V_{\text {int }}(\mathbf{r})$ is the interatomic interaction potential. Collisions in the $s$-wave channel are described by the regularized pseudopotential $V_{\text {int }}(\mathbf{r})=$ $\left(2 \pi \hbar^{2} a / m_{\text {red }}\right) \delta(\mathbf{r})(\partial / \partial r) r$, with $a$ the scattering length and $m_{\text {red }}$ the reduced mass [72]. Crucially, Eq. (6) replaces a complex many-body expectation value with a determinant over single-particle states, allowing efficient computation of a temperature estimate from the experimental data.

Decoherence in a homogeneous gas.-From here on, we focus on a three-dimensional (3D), homogeneous Fermi gas $\left(V_{\text {ext }}=0\right)$ of mean density $\bar{n}$ that is trapped in a box large enough to prevent finite-size effects. We assume the impurity is tightly confined so that the infinite-mass approximation is valid, i.e., $|\chi(\mathbf{r})|^{2} \approx \delta(\mathbf{r})$ and $m_{\text {red }}=m$. Analytical solutions for the single-particle wave functions are available in this case [63]; see the Supplemental Material for details of numerical calculations as well as an analytical treatment of the weak-coupling limit [73]. The physical scales of the gas are determined by the Fermi wave vector $k_{F}=\left(6 \pi^{2} \bar{n}\right)^{1 / 3}$, energy $E_{F}=\hbar^{2} k_{F}^{2} / 2 m$, time $\tau_{F}=\hbar / E_{F}$, and temperature $T_{F}=E_{F} / k_{B}$, while the dimensionless parameter $k_{F} a$ quantifies the impurity-gas coupling. The time evolution of the magnitude of the decoherence function for this system is shown in Fig. 2 for various coupling strengths and temperatures. We also plot the corresponding finite-temperature absorption spectra, which are related to $v(t)$ by a Fourier transform

$$
A(\omega)=\pi^{-1} \operatorname{Re} \int_{0}^{\infty} d t e^{-i \omega t} v(t) .
$$

Note that $A(\omega)$ is equivalent to the probability distribution of work performed by suddenly switching on the impurity potential $V_{\text {imp }}(\mathbf{r})[59,77,78]$. Since the properties of $v(t)$ and $A(\omega)$ have been extensively discussed in the literature $[24,56,62,63,79-81]$, here we simply summarize the notable features.

Scattering from the impurity generates particle-hole excitations in the gas. For weak coupling and low temperature, these excitations are initially limited to the vicinity of the Fermi surface due to Pauli blocking (see Fig. 1), but repeated scattering events eventually reorganize the entire Fermi sea: this is the essence of the OC [56]. Figure 2 shows that at relatively short times $\tau_{F}<t \ll \hbar \beta$, the OC manifests itself in a universal decoherence function $v(t) \sim e^{i w t} t^{-\left(\delta_{F} / \pi\right)^{2}}$, where $\delta_{F}=-\arctan \left(k_{F} a\right)$ is the scattering phase at the Fermi surface and $\hbar w$ is a collisional 


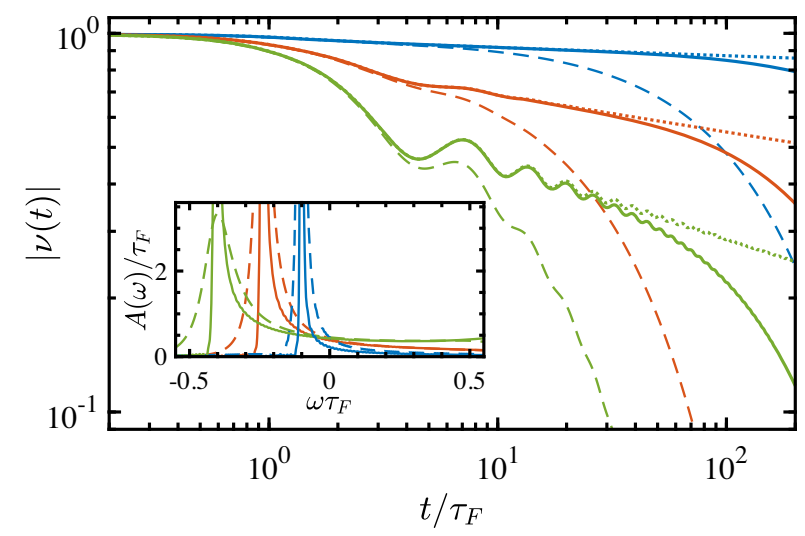

FIG. 2. Decoherence functions (main) and absorption spectra (inset) for the homogeneous gas, with coupling $k_{F} a=-0.5$ (blue), $k_{F} a=-1.5$ (red), and $k_{F} a=-6$ (green); and temperature $T=0$ (dotted), $T=0.01 T_{F}$ (solid), and $T=0.1 T_{F}$ (dashed). Spectra for $T=0$ not shown.

shift of the impurity's energy levels [63]. This short-time behavior is essentially dictated by the high-frequency tails of $A(\omega)$, which describe collective excitations of the whole Fermi sea and thus are largely insensitive to temperature. At later times, $v(t)$ departs from the zero-temperature behavior, decaying exponentially with a temperaturedependent rate for $t \gtrsim \hbar \beta$. This long-time behavior is determined by low-energy excitations close to the Fermi surface whose distribution is highly temperature dependent. This is seen in the dominant feature of the absorption spectra near $\omega=\Delta E$ where the zero-temperature edge singularity [79], resulting from the discontinuous Fermi surface, is softened at finite temperature into a broad peak (see Fig. 2 inset). The width of the peak is determined by both the temperature and the scattering length: larger values of $\left|k_{F} a\right|$ lead to a broader peak and thus a faster onset of exponential decay in the time domain.

Thermometric performance.-We now turn to the metrological implications of these features. Figure 3 shows the QSNR as a function of time and temperature for $k_{F} a=-0.5$. At a given temperature, the optimal measurement time corresponds to the maximum sensitivity, i.e., $\mathcal{Q}_{\max }=\max _{t} \mathcal{Q}(t)=\mathcal{Q}\left(t_{\max }\right)$, which shifts to progressively later times as the temperature decreases. We find that

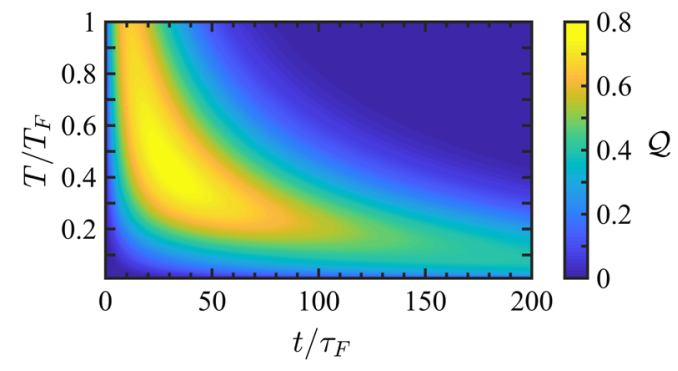

FIG. 3. QSNR as a function of temperature and evolution time for $k_{F} a=-0.5$. the maximum QSNR, shown by the large yellow region in Fig. 3, coincides with the relevant temperature range for current experiments [30,54,82], i.e., $T \gtrsim 0.1 T_{F}$, and good precision is retained down to the deeply degenerate regime. For example, with a coupling strength of $k_{F} a=-0.5$ and a temperature of $T=0.1 T_{F}$ we find $\mathcal{Q}_{\max } \approx 0.45$, meaning that an error of $\Delta T / T=10 \%$ can be achieved with $N \approx 500$ measurements after a time $t_{\max } \approx 150 \tau_{F}$, which is on the order of milliseconds for typical experimental parameters. This is eminently feasible, since a single gas sample may include thousands of independent impurities [54] and have a lifetime of several seconds [16,29].

Naturally, the maximum precision depends on the coupling strength. In Fig. 4(a) we show the dynamical QSNR for various scattering lengths finding, remarkably, that weaker coupling enhances thermometric performance. This can be understood by virtue of Eq. (4), which shows that probe states with high purity, i.e., large $|v|$, have a larger QFI. Since a state with high purity may have a sharply peaked distribution of measurement outcomes, a small parameter change is statistically easier to distinguish. Weak coupling is then preferable in light of the slower initial power-law decoherence-due ultimately to Pauli exclusion reducing the available phase space for scattering - which maintains purer, and therefore more sensitive, probe states. This is also illustrated in Fig. 4(b), which
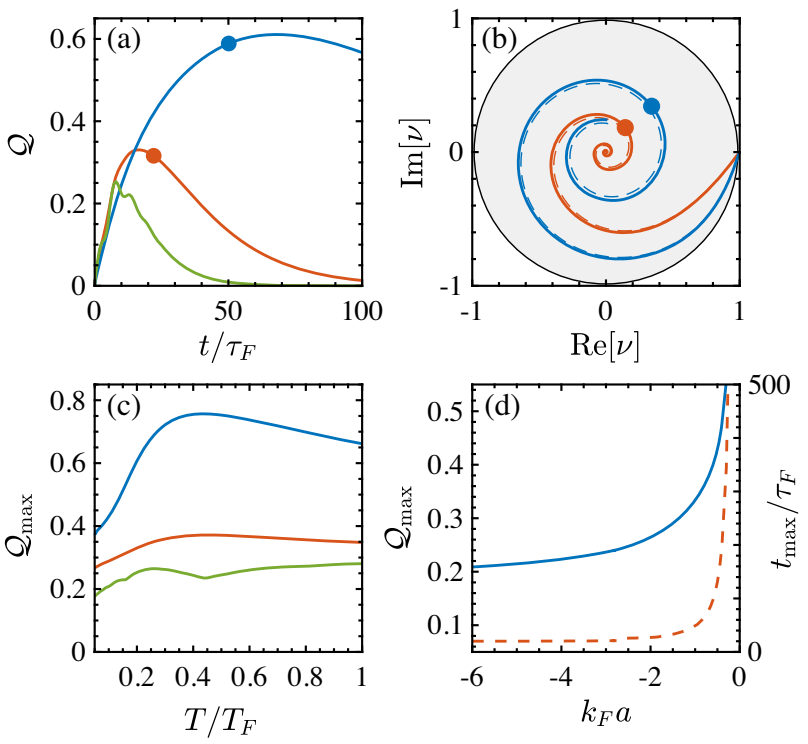

FIG. 4. (a) QSNR at $T=0.2 T_{F}$ as a function of time for $k_{F} a=$ -0.5 (blue), $k_{F} a=-1.5$ (red) and $k_{F} a=-6 \quad$ (green). (b) Decoherence function on the equator of the Bloch sphere for $T=0.2 T_{F}$ (solid lines) and $T=0.22 T_{F}$ (dashed lines) with $k_{F} a=-0.5$ (blue) and $k_{F} a=-1.5$ (red). Solid circles highlight the same instants in time in both panels. (c) Maximum sensitivity, $\mathcal{Q}_{\text {max }}$, as a function of temperature for $k_{F} a=-0.5$ (blue), $k_{F} a=$ -1.5 (red) and $k_{F} a=-6$ (green). (d) $\mathcal{Q}_{\max }$ (solid line) and corresponding measurement time (dashed line) as a function of coupling strength for $T=0.1 T_{F}$. See text for discussion. 
shows the path traced by the Bloch vector for two nearby temperatures and two coupling strengths. Clearly, weaker coupling ensures that the probe maintains larger purities and consequently is more sensitive to small temperature changes. In Fig. 4(c) we show that $\mathcal{Q}_{\max }$ is always larger for smaller scattering strengths, indicating that this qualitative picture holds for all temperatures.

However, this improved precision comes at the cost of measurement time. Indeed, from Fig. 2 we know that the onset of thermal behavior is delayed by weak coupling. We quantitatively examine the thermometric implications of this in Fig. 4(d) where we find that both $\mathcal{Q}_{\max }$ and $t_{\max }$ diverge as $\left|k_{F} a\right| \rightarrow 0$. In this limit, the universal exponent determining the decoherence rate is $\left(\delta_{F} / \pi\right)^{2}=\mathcal{O}\left(\left(k_{F} a\right)^{2}\right)$, whereas the phase evolves as $\phi=w t$, with $w=\mathcal{O}\left(k_{F} a\right)$ [73]. The QFI [Eq. (4)] is thus dominated by $\mathcal{F}_{T}^{\perp}$ while the SLD [Eq. (5)] is approximately $\hat{\Lambda}_{T} \approx \hat{\sigma}_{\perp}$, corresponding to a phase estimation protocol $[65,66]$. Since $w$ is temperature dependent, small temperature variations develop over time into large, distinguishable phase differences, resulting in the asymptotic scalings $t_{\max } \sim\left|k_{F} a\right|^{-2}$ and $\mathcal{Q}_{\max } \sim\left|k_{F} a\right|^{-1}$ at weak coupling [73]. The universal OC physics is therefore crucial because slow, algebraic decoherence allows a long time for phase accumulation without sacrificing the purity of the probe state.

Discussion.-Homogeneous ultracold gases represent a challenge for in situ thermometry, necessitating destructive time-of-flight measurements $[13,16]$. In fermionic systems this problem is exacerbated because the Pauli exclusion principle restricts thermal excitations to a small energy window near the Fermi surface, meaning that density measurements of any kind provide little information on temperature. In contrast, our proposal to infer temperature from decoherence is designed to exploit this structure of the Fermi sea. Specifically, exclusion effects slow the decay of the impurity decoherence function, allowing for enhanced sensitivity. Moreover, our scheme is inherently nonequilibrium, thus alleviating the need for thermalization of the probe before accurate temperature estimation is feasible.

The sensitivity of our probe can be controlled by using a Feshbach resonance to change the scattering length. Remarkably, we have shown that the highest QSNR is obtained for weak coupling, in contrast with the sensitivity enhancement found for thermalizing probes at strong coupling [83]. Practically speaking, weak coupling reduces the number of measurements needed to achieve a given precision, albeit at the cost of increasing the measurement time (and vice versa). This tunability allows the protocol to be optimized depending on the experimental constraints at hand. It is worth noting that the impurity decoherence function exhibits a universal dependence on a small number of parameters, $k_{F} a, E_{F}$, and $T$, which can each be determined via a similar interferometric protocol. For example, either $k_{F} a$ or $E_{F}$ can be determined from the temperature-independent behavior of $v(t)$ at short times.
This may assist calibration of the thermometer and obviates the need to incorporate independent measurements of the density or scattering length-with their associated experimental uncertainties - into the parameter estimation procedure.

Our analysis focused on homogeneous gases where conventional in situ thermometry is difficult. However, the same approach could in principle be applied to trapped gases with arbitrary geometry. In the Supplemental Material [73], we consider thermometry of the one-dimensional (1D) Fermi gas, finding similar sensitivities to the 3D case. Interestingly, the norm of the decoherence function is similar for homogeneous and harmonically trapped 1D gases (for $\omega_{0} t \ll \pi$, with $\omega_{0}$ the trap frequency [58]). However, the complex phase of $v(t)$ is significantly modified by the presence of the harmonic trap. Since this phase is sensitive to temperature, an optimally precise temperature estimator for a harmonically confined gas should account for the trap configuration. We emphasize that our theory based on Eq. (6) is computationally efficient for any size and geometry, requiring only the single-particle wave functions.

In summary, we have proposed a minimally destructive and local thermometry protocol based on the decoherence of immersed impurities, which offers a solution to the challenge of in situ thermometry for homogeneous Fermi gases. This complements recently developed techniques based on two-photon spectroscopy $[84,85]$. Future work could address the effect of impurity motion $[86,87]$ and correlations between probes generated via their mutual interaction with the gas $[25,88,89]$.

We are grateful to Alessandro Silva for unwittingly suggesting a cumulant expansion approach, and we thank Luis Correa, Michael Knap, Matteo Paris, Meera Parish, Patrick Potts, Yuval Sagi, and Artur Widera for useful comments on the manuscript. We acknowledge support from the European Research Council Starting Grant ODYSSEY (G. A. 758403), the JSPS KAKENHI18K13507, the SFI-Royal Society University Research Fellowship scheme, the Science Foundation Ireland Starting Investigator Research Grant "SpeedDemon" (No. 18/SIRG/5508), and the Okinawa Institute of Science and Technology Graduate University.

*mark.mitchison@tcd.ie †'gooldj@tcd.ie

[1] W. Hofstetter and T. Qin, J. Phys. B 51, 082001 (2018).

[2] L. Tarruell and L. Sanchez-Palencia, C. R. Phys. 19, 365 (2018).

[3] N. Navon, S. Nascimbène, F. Chevy, and C. Salomon, Science 328, 729 (2010).

[4] M. J. H. Ku, A. T. Sommer, L. W. Cheuk, and M. W. Zwierlein, Science 335, 563 (2012). 
[5] M. Inguscio, W. Ketterle, and C. Salomon, Proceedings of the International School of Physics "Enrico Fermi" 164, 1 (2008), https://www.iospress.nl/book/ultra-cold-fermi-gases/.

[6] L. E. Sadler, J. M. Higbie, S. R. Leslie, M. Vengalattore, and D. M. Stamper-Kurn, Nature (London) 443, 312 (2006).

[7] S. Hofferberth, I. Lesanovsky, B. Fischer, T. Schumm, and J. Schmiedmayer, Nature (London) 449, 324 (2007).

[8] S. Trotzky, Y.-A. Chen, A. Flesch, I. P. McCulloch, U. Schollwck, J. Eisert, and I. Bloch, Nat. Phys. 8, 325 (2012).

[9] M. Gring, M. Kuhnert, T. Langen, T. Kitagawa, B. Rauer, M. Schreitl, I. Mazets, D. A. Smith, E. Demler, and J. Schmiedmayer, Science 337, 1318 (2012).

[10] M. Cheneau, P. Barmettler, D. Poletti, M. Endres, P. Schauß, T. Fukuhara, C. Gross, I. Bloch, C. Kollath, and S. Kuhr, Nature (London) 481, 484 (2012).

[11] J.-P. Brantut, C. Grenier, J. Meineke, D. Stadler, S. Krinner, C. Kollath, T. Esslinger, and A. Georges, Science 342, 713 (2013).

[12] S. Krinner, T. Esslinger, and J.-P. Brantut, J. Phys. Condens. Matter 29, 343003 (2017).

[13] A. L. Gaunt, T. F. Schmidutz, I. Gotlibovych, R. P. Smith, and Z. Hadzibabic, Phys. Rev. Lett. 110, 200406 (2013).

[14] T. F. Schmidutz, I. Gotlibovych, A. L. Gaunt, R. P. Smith, N. Navon, and Z. Hadzibabic, Phys. Rev. Lett. 112, 040403 (2014).

[15] N. Navon, A. L. Gaunt, R. P. Smith, and Z. Hadzibabic, Science 347, 167 (2015).

[16] B. Mukherjee, Z. Yan, P. B. Patel, Z. Hadzibabic, T. Yefsah, J. Struck, and M. W. Zwierlein, Phys. Rev. Lett. 118, 123401 (2017).

[17] K. Hueck, N. Luick, L. Sobirey, J. Siegl, T. Lompe, and H. Moritz, Phys. Rev. Lett. 120, 060402 (2018).

[18] B. Mukherjee, P. B. Patel, Z. Yan, R. J. Fletcher, J. Struck, and M. W. Zwierlein, Phys. Rev. Lett. 122, 203402 (2019).

[19] Z. Yan, P. B. Patel, B. Mukherjee, R. J. Fletcher, J. Struck, and M. W. Zwierlein, Phys. Rev. Lett. 122, 093401 (2019).

[20] G. Roati, F. Riboli, G. Modugno, and M. Inguscio, Phys. Rev. Lett. 89, 150403 (2002).

[21] C. Silber, S. Günther, C. Marzok, B. Deh, P. W. Courteille, and C. Zimmermann, Phys. Rev. Lett. 95, 170408 (2005).

[22] A. N. Wenz, G. Zurn, S. Murmann, I. Brouzos, T. Lompe, and S. Jochim, Science 342, 457 (2013).

[23] P. Massignan, M. Zaccanti, and G. M. Bruun, Rep. Prog. Phys. 77, 034401 (2014).

[24] R. Schmidt, M. Knap, D. A. Ivanov, J.-S. You, M. Cetina, and E. Demler, Rep. Prog. Phys. 81, 024401 (2018).

[25] D. Hangleiter, M. T. Mitchison, T. H. Johnson, M. Bruderer, M. B. Plenio, and D. Jaksch, Phys. Rev. A 91, 013611 (2015).

[26] L. A. Correa, M. Mehboudi, G. Adesso, and A. Sanpera, Phys. Rev. Lett. 114, 220405 (2015).

[27] M. Mehboudi, A. Lampo, C. Charalambous, L. A. Correa, M. A. García-March, and M. Lewenstein, Phys. Rev. Lett. 122, 030403 (2019).

[28] C. Regal, Experimental realization of BCS-BEC crossover physics with a Fermi gas of atoms, Ph.D. thesis, University of Colorado, 2005.

[29] F. M. Spiegelhalder, A. Trenkwalder, D. Naik, G. Hendl, F. Schreck, and R. Grimm, Phys. Rev. Lett. 103, 223203 (2009).
[30] S. Nascimbène, N. Navon, K. J. Jiang, F. Chevy, and C. Salomon, Nature (London) 463, 1057 (2010).

[31] D. McKay and B. DeMarco, New J. Phys. 12, 055013 (2010).

[32] R. Olf, F. Fang, G. E. Marti, A. MacRae, and D. M. Stamper-Kurn, Nat. Phys. 11, 720 (2015).

[33] M. Hohmann, F. Kindermann, T. Lausch, D. Mayer, F. Schmidt, and A. Widera, Phys. Rev. A 93, 043607 (2016).

[34] R. S. Lous, I. Fritsche, M. Jag, B. Huang, and R. Grimm, Phys. Rev. A 95, 053627 (2017).

[35] M. G. A. Paris, J. Phys. A 49, 03LT02 (2016).

[36] A. D. Pasquale, D. Rossini, R. Fazio, and V. Giovannetti, Nat. Commun. 7, 12782 (2016).

[37] K. V. Hovhannisyan and L. A. Correa, Phys. Rev. B 98, 045101 (2018).

[38] S. Campbell, M. G. Genoni, and S. Deffner, Quantum Sci. Technol. 3, 025002 (2018).

[39] P. P. Potts, J. B. Brask, and N. Brunner, Quantum 3, 161 (2019).

[40] M. R. Jørgensen, P. P. Potts, M. G. A. Paris, and J. B. Brask, arXiv:2001.04096.

[41] G. Ferrari, Phys. Rev. A 59, R4125 (1999).

[42] S. Jevtic, D. Newman, T. Rudolph, and T. M. Stace, Phys. Rev. A 91, 012331 (2015).

[43] L. Mancino, M. Sbroscia, I. Gianani, E. Roccia, and M. Barbieri, Phys. Rev. Lett. 118, 130502 (2017).

[44] P. P. Hofer, J. B. Brask, M. Perarnau-Llobet, and N. Brunner, Phys. Rev. Lett. 119, 090603 (2017).

[45] V. Cavina, L. Mancino, A. De Pasquale, I. Gianani, M. Sbroscia, R. I. Booth, E. Roccia, R. Raimondi, V. Giovannetti, and M. Barbieri, Phys. Rev. A 98, 050101(R) (2018).

[46] Q. Bouton, J. Nettersheim, D. Adam, F. Schmidt, D. Mayer, T. Lausch, E. Tiemann, and A. Widera, Phys. Rev. X 10, 011018 (2020).

[47] M. Bruderer and D. Jaksch, New J. Phys. 8, 87 (2006).

[48] C. Sabín, A. White, L. Hackermuller, and I. Fuentes, Sci. Rep. 4, 6436 (2015).

[49] T. H. Johnson, F. Cosco, M. T. Mitchison, D. Jaksch, and S. R. Clark, Phys. Rev. A 93, 053619 (2016).

[50] S. Razavian, C. Benedetti, M. Bina, Y. Akbari-Kourbolagh, and M. G. A. Paris, Eur. Phys. J. Plus 134, 284 (2019).

[51] D. C. McKay and B. DeMarco, Rep. Prog. Phys. 74, 054401 (2011).

[52] R. Onofrio, Phys. Usp. 59, 1129 (2016).

[53] M. Cetina, M. Jag, R. S. Lous, J. T. M. Walraven, R. Grimm, R. S. Christensen, and G. M. Bruun, Phys. Rev. Lett. 115, 135302 (2015).

[54] M. Cetina, M. Jag, R. S. Lous, I. Fritsche, J. T. M. Walraven, R. Grimm, J. Levinsen, M. M. Parish, R. Schmidt, M. Knap, and E. Demler, Science 354, 96 (2016).

[55] G. Ness, C. Shkedrov, Y. Florshaim, and Y. Sagi, arXiv:2001.10450.

[56] P. W. Anderson, Phys. Rev. Lett. 18, 1049 (1967).

[57] T. Fogarty, S. Deffner, T. Busch, and S. Campbell, Phys. Rev. Lett. 124, 110601 (2020).

[58] A. Sindona, J. Goold, N. Lo Gullo, S. Lorenzo, and F. Plastina, Phys. Rev. Lett. 111, 165303 (2013).

[59] A. Sindona, J. Goold, N. L. Gullo, and F. Plastina, New J. Phys. 16, 045013 (2014). 
[60] F. Cosco, M. Borrelli, E.-M. Laine, S. Pascazio, A. Scardicchio, and S. Maniscalco, New J. Phys. 20, 073041 (2018).

[61] F. Tonielli, R. Fazio, S. Diehl, and J. Marino, Phys. Rev. Lett. 122, 040604 (2019).

[62] J. Goold, T. Fogarty, N. Lo Gullo, M. Paternostro, and T. Busch, Phys. Rev. A 84, 063632 (2011).

[63] M. Knap, A. Shashi, Y. Nishida, A. Imambekov, D. A. Abanin, and E. Demler, Phys. Rev. X 2, 041020 (2012).

[64] C. Chin, R. Grimm, P. Julienne, and E. Tiesinga, Rev. Mod. Phys. 82, 1225 (2010).

[65] M. G. A. Paris, Int. J. Quantum. Inform. 07, 125 (2009).

[66] G. Tóth and I. Apellaniz, J. Phys. A 47, 424006 (2014).

[67] M. Mehboudi, A. Sanpera, and L. A. Correa, J. Phys. A 52, 303001 (2019).

[68] S. L. Braunstein and C. M. Caves, Phys. Rev. Lett. 72, 3439 (1994).

[69] W. Zhong, Z. Sun, J. Ma, X. Wang, and F. Nori, Phys. Rev. A 87, 022337 (2013).

[70] D. A. Abanin and L. S. Levitov, Phys. Rev. Lett. 94, 186803 (2005).

[71] N. d'Ambrumenil and B. Muzykantskii, Phys. Rev. B 71, 045326 (2005).

[72] T. Busch, B.-G. Englert, K. Rzażewski, and M. Wilkens, Found. Phys. 28, 549 (1998).

[73] See Supplemental Material at http://link.aps.org/ supplemental/10.1103/PhysRevLett.125.080402 for details of the numerical calculations, analytical descriptions of decoherence and thermometric precision in the weakcoupling limit, and an analysis of dephasing thermometry in a 1D gas, including citations to Refs. [74-76].
[74] R. Kubo, J. Phys. Soc. Jpn. 17, 1100 (1962).

[75] K. E. Cahill and R. J. Glauber, Phys. Rev. A 59, 1538 (1999).

[76] M. Olshanii, Phys. Rev. Lett. 81, 938 (1998).

[77] A. Silva, Phys. Rev. Lett. 101, 120603 (2008).

[78] T. Keller and T. Fogarty, Phys. Rev. A 94, 063620 (2016).

[79] P. Noziéres and C. T. De Dominicis, Phys. Rev. 178, 1097 (1969).

[80] W. E. Liu, Z.-Y. Shi, J. Levinsen, and M. M. Parish, Phys. Rev. Lett. 125, 065301 (2020).

[81] W. E. Liu, Z.-Y. Shi, M. M. Parish, and J. Levinsen, Phys. Rev. A 102, 023304 (2020)..

[82] S. Nascimbène, N. Navon, K. J. Jiang, L. Tarruell, M. Teichmann, J. McKeever, F. Chevy, and C. Salomon, Phys. Rev. Lett. 103, 170402 (2009).

[83] L. A. Correa, M. Perarnau-Llobet, K. V. Hovhannisyan, S. Hernández-Santana, M. Mehboudi, and A. Sanpera, Phys. Rev. A 96, 062103 (2017).

[84] I. Gotlibovych, T. F. Schmidutz, A. L. Gaunt, N. Navon, R. P. Smith, and Z. Hadzibabic, Phys. Rev. A 89, 061604(R) (2014).

[85] C. Shkedrov, G. Ness, Y. Florshaim, and Y. Sagi, Phys. Rev. A 101, 013609 (2020).

[86] M. M. Parish and J. Levinsen, Phys. Rev. B 94, 184303 (2016).

[87] W. E. Liu, J. Levinsen, and M. M. Parish, Phys. Rev. Lett. 122, 205301 (2019).

[88] M. T. Mitchison, T. H. Johnson, and D. Jaksch, Phys. Rev. A 94, 063618 (2016).

[89] G. Planella, M. Mehboudi, and A. Acín, arXiv:2001.11812. 